\title{
Paraplegia Aguda. Uma Complicação Rara da Dissecção Aórtica
}

\author{
Rena to Côrtes de Lacerda, Patrícia Bernard Andrea, Evaristo Machado N eto, Carlos Baptista de \\ Figueiredo, A lfredo Brasil Teixeira, Paulo Cézar Monteiro de Carvalho \\ Rio de Janeiro, RJ
}

\begin{abstract}
Mulher normotensa de 67 anos desenvolveu síncope, seguida de choque, e, mesmo após estabilização hemodinâmica, permaneceu anúrica. Observaram-se paraplegia e paresia do membro superior direito, além de isquemia das extremidades distais dos membros inferiores. A hipótese de dissecção aórtica aguda foi considerada e confirmada através de tomografia computadorizada, tendo sido a paraplegia atribuída a infarto isquêmico da medula espinhal. Óbito ocorreu no $4^{\circ}$ dia de evolução, conseqüente a tamponamento pericárdico. São revistas as dificuldades diagnósticas relacionadas a manifestações pouco conhecidas da dissecção aórtica, enfatizandose, como complicação rara, a paraplegia aguda.
\end{abstract}

\section{Acute Paraplegia.A Rare Complication of Aortic Dissection}

A 67 year-old normotensive woman had a syncope followed by shock and remained anuric after hemodynamic stabilization. Paraplegia and paresis of the right upper limb, as well as signs of ischemia of the distal lower limbs were noted. The possibility of acute aortic dissection was raised and confirmed by computed tomography. The paraplegia was attributed to an ischemic infarction of the spinal cord. The patient died on the fourth hospital day due to a pericardial tamponade. This rare and not well recognized complication of aortic dissection is briefly reviewed.

Arq Bras Cardiol, volume 70 (n 4), 275-278, 1998

A dissecção aórtica aguda (DAA) é uma emergência médica que exige pronto diagnóstico e terapêutica agressiva, já que as complicações a ela associadas são múltiplas, graves e freqüentemente fatais ${ }^{1}$. Quando o paciente apresenta-se com dor precordial ou tóraco-abdominal de início abrupto, hipertensão grave e eletrocardiograma (ECG) não sugestivo de infarto agudo do miocárdio (IAM), a hipótese diagnóstica geralmente é levantada, sobretudo se a radiografia do tórax mostrar alargamento do mediastino ${ }^{2,3}$, devendo-se prosseguir com outros métodos de imagem de comprovada eficácia diagnóstica ${ }^{4-6}$, conforme a disponibilidade dos recursos locais. A DAA, entretanto, pode se manifestar de outras formas, e seu diagnóstico ficar mascarado, devendo-se estar muito atento para alguma de suas apresentações menos comuns: insuficiência aórtica aguda, levando à insuficiência cardíaca ou choque, tamponamento cardíaco, síncope ou choque não esclarecido, acidente

Hospital Geral de Bonsucesso - Rio de Janeiro

Correspondência: Renato Côrtes de Lacerda - Rua Farme de Amoedo, 82/701 22420-020 - Rio de Janeiro, RJ

Recebido para publicação em 21/10/97

Aceito em 15/1/98 vascular encefálico (AVE), isquemia aguda de membros inferiores e IAM ${ }^{1,7-9}$. Relatamos um caso que apresenta complicação rara e pouco conhecida da DAA, a paraplegia aguda.

\section{Relato do Caso}

Mulher de 67 anos, branca, apresentou quadro de síncope, sendo levada por familiares a um Pronto-Socorro, onde foi atendida com quadro de choque e transferida no dia seguinte para a Unidade de Cardiologia Intensiva do Hospital Geral de Bonsucesso. Encontrava-se torporosa, dispnéica, extremidades levemente cianóticas e frias, taquicárdica (130bpm), pressão arterial (PA) sistólica $60 \mathrm{mmHg}$. Medicada com oxigênio por cânula nasal, solução fisiológica, dopamina e dobutamina, apresentou melhora hemodinâmica significativa, possibilitando a suspensão das drogas vasoativas após $24 \mathrm{~h}$, mantendo PA em torno de $110 / 70 \mathrm{mmHg}$ em ambos os membros superiores. Obteve-se informação de que levava vida ativa como dona de casa e de que, dois anos antes, fora tratada de um câncer uterino, através de radioterapia, sem que tivesse sido submetida a histerectomia. Não era hipertensa e desconhecia ser portadora de doença cardíaca. 


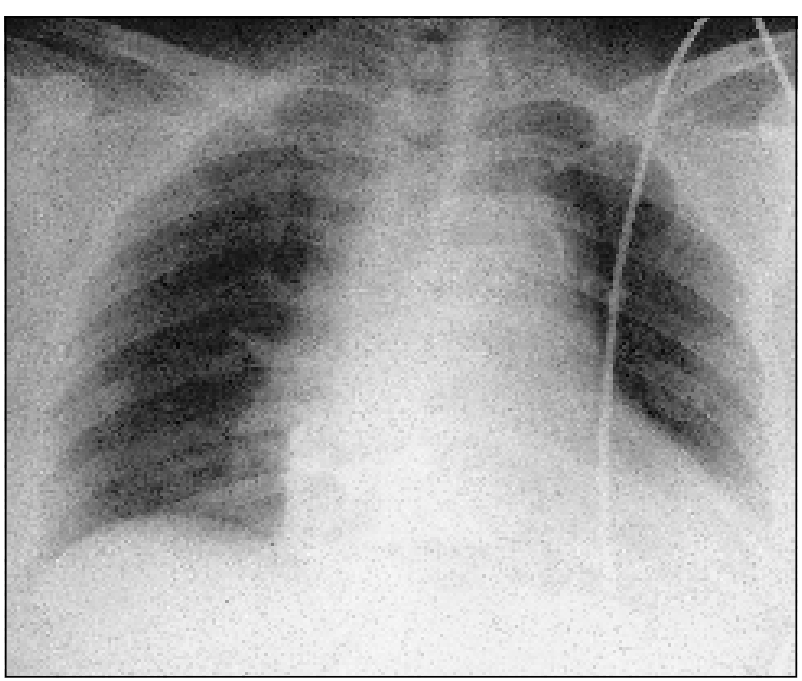

Fig. 1 - Radiografia do tórax em AP no leito, mostrando cardiomegalia e alargamento do mediastino, com calcificação na croça da aorta a $2 \mathrm{~cm}$ do bordo externo do vaso, sugerindo separação entre a íntima e a adventícia (sinal do cálcio).

Após estabilização hemodinâmica, continuou sonolenta, desorientada no tempo e no espaço. Não havia rigidez de nuca, as pupilas eram isocóricas e fotorreagentes e o exame dos pares cranianos não mostrou anormalidade. Observaram-se paresia do membro superior direito e paraplegia, com perda da sensibilidade no abdômen e membros inferiores. Os pulsos carotídeos eram amplos, assim como os pulsos dos membros superiores, femorais e poplíteos, e não havia sopro nos trajetos arteriais. Os pulsos pediosos e tibiais posteriores estavam impalpáveis. Os membros inferiores estavam frios no seu terço inferior e tornaram-se progressivamente cianóticos. Permanecia taquicárdica (120bpm). Não foram auscultados sopros nem atrito pericárdico e as bulhas cardíacas eram normofonéticas. As jugulares eram visíveis a $30^{\circ}$ e a pressão venosa central estava em $20 \mathrm{~cm}$ de $\mathrm{H}_{2} \mathrm{O}$. O murmúrio vesicular estava diminuído nas bases pulmonares. O abdômen era flácido e indolor, sem massas ou vísceras palpáveis.

ECG mostrava taquicardia sinusal e alterações de ST e $\mathrm{T}$ inespecíficas. A radiografia de tórax, obtida no leito, era sugestiva de cardiomegalia e alargamento do mediastino, observando-se ainda ateroma calcificado na croça da aorta, projetando-se a $2 \mathrm{~cm}$ do bordo externo do vaso, sugerindo dupla luz aórtica (fig. 1).

Foram realizadas ultra-sonografia abdominal, ecocolor-Doppler transtorácico e tomografia computadorizada (TC) do tórax e abdômen com contraste (fig. 2). Esses exames demonstraram a presença de dissecção aórtica, iniciando-se na aorta ascendente, progredindo pelo arco aórtico, aorta descendente e abdominal até abaixo das artérias renais, não ficando, entretanto, evidenciado o ponto de início da dissecção. O tronco braquiocefálico, a carótida comum esquerda e a subclávia esquerda não pareciam estar comprometidos pela dissecção. Observou-se leve derrame pericárdico, não havendo contudo insuficiência aórtica. Uma TC do crânio com contraste não mostrou anormalidades.

A PA subiu, no dia seguinte à internação, a 140/
$80 \mathrm{mmHg}$, permanecendo contudo anúrica, sem responder a furosemida intravenosa. A partir de então foi medicada com nitroprussiato de sódio e metoprolol, por via intravenosa, estabilizando-se a PA abaixo de $120 / 80 \mathrm{mmHg}$ e normalizando a frequiência cardíaca. O hematócrito inicial era $39 \%$ e os leocócitos 15.800, valores que caíram, nos dias subseqüentes, para $33 \%$ e 10.600 . Os níveis de uréia e creatinina, inicialmente $87 \mathrm{mg} \%$ e $3,7 \mathrm{mg} \%$, elevaram-se progressivamente, chegando a $235 \mathrm{mg} \%$ e $7,6 \mathrm{mg} \%$, respectivamente. Observou-se também uma intensa elevação da creatinofosfoquinase (CPK), partindo de 8.438U no dia da internação, chegando a 46.222U dois dias após. A fração MB (CKMB) subiu proporcionalmente menos, indo de $186 \mathrm{U}$ a $435 \mathrm{U}$ no mesmo período. A aspartato transaminase atingiu um pico elevadíssimo de 7.042U, enquanto a desidrogenase lática
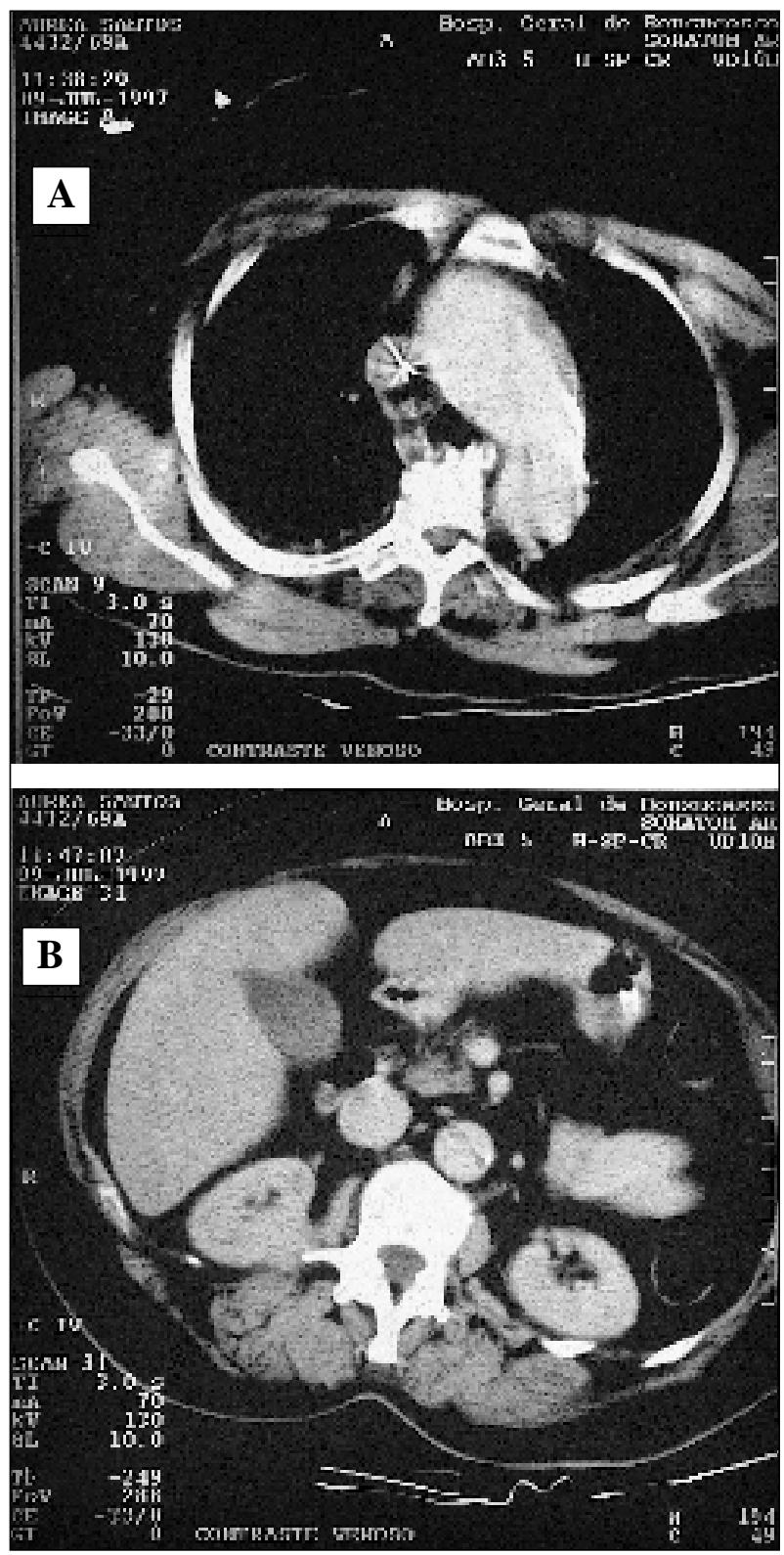

Fig. 2 - Observa-se imagem linear hipodensa, típica de dissecção arterial, estendendo-se desde a aorta proximal (A) até a emergência das artérias renais(B); Nota-se ausência de excreção renal de contraste sugerindo dissecção das artérias renais(B). 
(DHL) foi até 2.465U. Os níveis de potássio sérico não foram além de $5,4 \mathrm{mEq} / \mathrm{L}$. Faleceu quatro dias após o quadro inicial de síncope, subitamente, após queda abrupta da PA.

\section{Discussão}

O diagnóstico de DAA pode ser muito difícil, quando suas manifestações clínicas fogem ao padrão habitual, a saber: 1) dor torácica ou tóraco-dorso-abdominal de início abrupto e caráter migratório; 2) hipertensão arterial grave;3) ECG não sugestivo de IAM; 4) radiografia de tórax mostrando alargamento do mediastino.

Na experiência da Clínica Mayo, nos Estados Unidos, com 236 casos de DAA, vistos entre 1980 e 1990, em apenas $62 \%$ dos casos a impressão clínica inicial foi de DAA, sendo que em $28 \%$ o diagnóstico só foi feito pós-morte ${ }^{1}$. Um estudo retrospectivo feito pela Universidade de Kioto, no Japão, verificou que, de um total de 41 pacientes com DAA, 18 haviam sido encaminhados àquela instituição com diagnósticos equivocados: dois como IAM não complicado (DAA obstruindo parcialmente o tronco da coronária esquerda), um como pneumonia (alargamento do mediastino interpretado incorretamente), dois como infarto cerebral (DAA ocluindo o tronco bráquio-encefálico), seis como abdômen agudo, um como colelitíase, cinco como isquemia dos membros inferiores e um como metástase pericárdica ${ }^{7}$.

No nosso caso, a paciente de 67 anos e sem histórico de hipertensão foi encaminhada à nossa instituição com o diagnóstico de choque, conseqüente a IAM. De fato, uma acentuada elevação da CPK, da DHL e das transaminases, respaldava essa hipótese, embora a relação CKMB/CPK fosse muito inferior à esperada em se tratando de IAM. Além disso o ECG mostrava alterações de ST e T pouco expressivas, não sugestivas de um IAM, que estivesse evoluindo com tanta repercussão hemodinâmica.

A observação de que apresentava paraplegia e paresia do membro superior direito fez suspeitar de um evento vascular agudo, já que a subitaneidade do quadro tornava improvável o diagnóstico de compressão medular, decorrente de processo expansivo neoplásico, relacionado ao câncer uterino diagnosticado e tratado por radioterapia dois anos antes. A possibilidade de oclusão aórtica aguda foi levantada. Essa catástrofe clínica rara pode ser conseqüente a trombose de uma aorta ateromatosa, a trombose de um aneurisma da aorta, ou a embolização de material aterosclerótico ${ }^{10}$, condições capazes de bloquear o fluxo sangüíneo para as artérias espinhais anteriores e posteriores, levando a isquemia ou infarto da medula espinhal. Nessas circunstâncias, contudo, normalmente se observa isquemia de ambos os membros inferiores ${ }^{10}$,e, embora a doente apresentasse sinais de isquemia no terço inferior dos membros inferiores, os pulsos femorais e poplíteos estavam preservados. Considerou-se então, o diagnóstico de DAA que, inclusive, poderia explicar a paresia do membro superior direito, admitindo-se que a paciente pudesse ter tido um AVE por dissecção da carótida esquerda. A radiografia de tórax, obtida em AP no leito (fig. 1), reforçou a impressão clínica de DAA, então confirmada através da TC (fig. 2), ultrasonografia e ecocardiograma-Doppler-transtorácico. A TC do crânio, normal, não excluiu a possibilidade de AVE isquêmico, já que freqüentemente o infarto cerebral sóé visto após alguns dias de evolução.

Uma revisão da literatura permitiu-nos encontrar algumas referências sobre paraplegia, conseqüente à DAA, a maioria sob forma de relato de caso, sendo que nenhum autor reuniu mais do que três pacientes em suas casuísticas ${ }^{11-18}$. A paraplegia é atribuída a isquemia ou infarto da medula espinhal devido a interrupção do fluxo sangüíneo para as artérias espinhais anteriores e posteriores. O infarto, tipicamente, ocorre na região compreendida entre a artéria de Adamkiewics (ramo da aorta abdominal que irriga a medula espinhal)e a artéria espinhal anterior (ramo das artérias vertebrais). Quase a metade dos pacientes faleceu na fase aguda da DAA, em alguns casos em decorrência de tamponamento cardíaco ${ }^{11-18}$. Alguns apresentaram reversão da paraplegia, ainda na fase aguda da DAA ${ }^{11,12,14,16} \mathrm{e}$, em outros, a paraplegia não foi acompanhada de nenhum tipo de dor ${ }^{12,16}$.

Alguns aspectos do caso devem ser comentados: 1) a dissecção das artérias renais foi a causa provável da insuficiência renal (fig. 2). O choque prolongado, acompanhado de lesão muscular e mioglobinúria (observar elevação de CPK), certamente também contribuíram;2) a isquemia de extremidades foi atribuída a possível embolização proveniente da extensa ateromatose calcificada da aorta e/ou ao estado de hipoperfusão tecidual prolongada; 3 ) a indicação de cirurgia foi evidentemente considerada, em se tratando de dissecção aórtica do tipo proximal, com graves complicações associadas, mas não pôde, ser concretizada por diversas razões; 4) o óbito da paciente foi súbito, precedido de choque, sendo atribuído a tamponamento pericárdico por ruptura da aorta para o saco pericárdico, hipótese bastante provável, tendo em vista que o ecocardiograma já havia demonstrado a presença de derrame pericárdico, sugerindo que já tivesse havido rompimento contido para o pericárdio.

\section{Referências}

1. Spittell PC, Spittell JA Jr, Joyce JW et al - Clinical features and differential diagnosis of aortic dissection: experience with 236 cases (1980 through 1990). Mayo Clin Proc 1993; 68: 642-51.

2. Luker GD, Glazer HS, Eagar G, GutierrezFR, Sagel SS - Aortic dissection: effect of prospective chest radiographic diagnosis on delay to definitive diagnosis. Radiology 1994; 193: 813-19.
3. Jagannah AS, Sos TA, Lockhart SH, Saddekni S, Sniderman KW - Aortic dissection: a statistical analysis of the usefulness of plain chest radiographic findings. Am J Roentgenol 1986; 147: 1123-6.

4. Vu FH, Young N, Soo YS - Imaging of thoracic aortic dissection. Australas Radiol 1994; 38: 170-5.

5. Laissy JP, Blanc F, Soyer P et al - Thoracic aortic dissection: diagnosis with 
transesophageal echocardiography versus MR imaging. Radiology 1995; 194: 331-6.

6. Fisher ER, Stern EJ, Godwin JD 2 $2^{\text {nd }}$, Otto CM, Johnson JA - Acute aortic dissection: typical and atypical imaging features. Radiographics 1994; 14: 1263-71.

7. Hashimoto S, Kumada T, Kubo S et al - The pitfalls in the clinical diagnosis of dissecting aortic aneurism. J Cardiol 1992; 22: 467-74.

8. Matsuo H - Pathophysiology and complications of aortic dissection. Nippon Geka Gakkai Zasshi 1996; 97: 879-83.

9. Murad H - Dissecção aórtica: diagnóstico e tratamento. Rev Soc Cardiol Est SP 1997; 10: 69-73.

10. Meagher AP, Lord RS, Graham AR, Hill DA - Acute aortic occlusion presenting with lower limb paralysis. J Cardiovasc Surg 1991; 32: 643-7.

11. Waltimo O, Karli P-Aortic dissection and paraparesis. Eur Neurol 1980; 19: 254-7.

12. Zull DN, Cydulka R - Acute paraplegia: a presenting manifestation of aortic dis- section. Am J Med 1998; 84: 765-70.

13. Kellet MW, Young GR, Fletcher NA - Paraparesis due to syphilitic aortic dissection. Neurology 1997; 48: 221-3.

14. Tanaka T, Uemura K, Sugiura Met al - Transient paraplegia caused by acute aortic dissection - case report. Neurol Med Chir 1990; 30: 54-8.

15. Kim SW, Kim RC, Choi BH, Gordon SK-Non-traumatic ischaemic myelopathy: a review of 25 cases. Paraplegia 1988; 26: 262-72.

16. Waltimo O, Karli P - Aortic dissection and paraparesis. Eur Neurol 1980; 19: 254-7.

17. Gascard E, Huguet JF, dor V, Rapuzzi A - Aortic dissection flaccid paraplegia. Mars Med 1970; 107: 421-6.

18. Krishnamurthy P, Chandrasekaran K, Rodriguez Vega JR, Grunewalk K - Acute thoracic aortic occlusion resulting from complex aortic dissection and presenting as paraplegia. J Thorac Imaging 1994; 9: 101-4. 